\title{
Exploring the Effects of Violating the 180-Degree Rule on Film Viewing Preferences
}

\author{
George Kachkovski \\ Daniil Vasilyev \\ Michael Kuk \\ Alan Kingstone* \\ Chris N. H. Street*
}

*Correspondence to

Prof. Alan Kingstone

Dr. Chris N. H. Street

Department of Psychology

Department of Psychology

University of British Columbia

University of Huddersfield

2329 West Mall

Queensgate

Vancouver, BC Canada

Huddersfield, West Yorkshire, UK

V6T $1 Z 4$

HD1 3DH

alan.kingstone@ubc.ca

c.street@hud.ac.uk

\section{Funding Statement}

This research was supported by grants to AK from the Natural Sciences and Engineering Research Council of Canada, and the Social Sciences and Humanities Research Council of Canada. 


\begin{abstract}
The 180-degree rule is thought to help smooth the change between film shots. When two individuals are speaking to each other, there is an imaginary axis of action running between them. If the camera crosses this axis, it breaks the 180-degree rule. A violation of the 180-degree rule is thought to have negative effects on viewers' enjoyment of films. The present study investigated this idea. Experiment 1 established that naive participants can detect violations in videos. Experiment 2 tested the putative negative effects of 180 -degree rule violations. The results indicated that violations can confuse and disorient viewers. Critically, as revealed by Experiment 3, violations did not alter the viewers' liking of a video: Viewers were as likely to prefer a video with a 180-degree violation as one without. Collectively, these data shed light on fundamental beliefs regarding the 180-degree rule, which may help inform filming decisions around film enjoyment.
\end{abstract}

Keywords: 180-degree rule; film editing; film enjoyment; continuity editing 
Exploring the Effects of Violating the 180-Degree Rule

on Film Viewing Preferences

A single scene in a movie consists of "shots" (single, uninterrupted recordings from a camera), with transitions between shots called "cuts". It is the filmmaker's task to assemble these shots in a meaningful way to create a narrative that is easy and entertaining to follow (Bordwell \& Thompson, 2012; Mascelli, 1965) ${ }^{1}$. To help attain this, filmmakers have created a series of film editing rules, collectively called "continuity editing" (Bordwell \& Thompson, 2012; Kraft, 1986). At the center of continuity editing is the 180-degree rule, violations of which are thought to result in potentially confusing or disorienting transitions (Bordwell \& Thompson, 2012; Hochberg \& Brooks, 1996; Proferes, 2008; Shimamura, 2013). Such discontinuous transitions are said to detract from the subjective experience of the film (see Hochberg \& Brooks, 1996; Kraft, Cantor, \& Gottdiener, 1991).

The 180-degree rule works as follows: A wide establishing shot is used to introduce the scene's location (with all of its entrances and exits) and to determine an "axis of action"- an imaginary line between two or more interacting characters - along which the action and dialogue happens (Bordwell \& Thompson, 2012; Giannetti, 2001; Smith \& Henderson, 2008). From that moment on, during the subsequent interaction the camera must remain on one side of the axis. For example, the director may begin cutting closer to the characters as they speak, all the while remaining on the same side of the axis. This continues until a new set of relationships

\footnotetext{
${ }^{1}$ Some filmmakers will purposely violate the 180-degree rule out of necessity, such as Murch (2001) who states that "[When choosing to make a cut,] if none of the other edits has the right emotion, then sacrificing spatial continuity is well worth it." Some filmmakers violate the rule to produce an effect, such as John Ford, who violates the rule "to enhance the tension in
} a...scene" (Shimamura, 2013). 
is established (e.g., by introducing a new establishing shot when another character enters the interaction). To cross the axis would be to violate the 180-degree rule.

As a result of adhering to the 180-degree rule, the characters remain on the left or right side of the frame throughout the interaction (Ascher \& Pincus, 2013; Bordwell \& Thompson, 2012; Giannetti, 2001; Proferes, 2008). If character A begins on the left side of the screen, they will remain on the left side of the screen. Similarly, if character A is looking towards the right side of the screen, their eye line will remain directed towards the right side of the screen.

The filmmaking community takes the position that violations of the 180-degree rule have negative consequences on the viewing experience (e.g., Ascher \& Pincus, 2013; Bordwell \& Thompson, 2012; Carroll, 1980; Giannetti, 2001; Murch, 2001; Proferes, 2008). For example, recent filmmaking books state that violations "will distract us from the unfolding plot" (Bordwell \& Thompson, 2012) and causes the "dynamics of the dramatic moment [to be] broken" (Proferes, 2008). These statements are somewhat open to interpretation but carry with them the implication that violations of the 180-degree rule negatively affect viewer's enjoyment.

The empirical psychological literature has focused more on the accuracy of a spatial representation of the scene than on viewer's enjoyment (Kraft, 1987; Kraft, Cantor \& Gottdiener, 1991). However, there is work demonstrating that because violating the 180 -degree rule flips characters' positions in relation to the camera, it disrupts the audience's understanding of the relative orientations on screen (Hochberg \& Brooks, 1996). This disorientation allegedly has a negative impact on the viewing experience (Hochberg \& Brooks, 1996; Kraft, Cantor, \& Gottdiener, 1991) because as the audience loses track of spatial positions on screen the emotion is lost (Proferes, 2008). The issue of whether violations affect viewing enjoyment, however, has limited objective testing or empirical support (see Shimamura, 2013). 
To our knowledge, no research to date has directly tested whether viewers can identify violations when they occur (see Levin \& Wang, 2009). Research on 180-degree violations has established that children (Frith \& Robson, 1975) and adults (Kraft et al., 1991) are less successful at remembering where objects were located in a scene if the cut violates the 180degree rule. This suggests that violations impact the spatial understanding of scenes (Huff \& Schwan, 2012). Convergent with this interpretation, eye movement studies reveal that viewers attempt to stitch together the spatial layout of the scene by finding common areas of the scene between two shots (Germeys \& d'Ydewalle, 2007; d'Ydewalle, Desmet, \& Van Rensbergen, 1998; see also Levin \& Wang, 2009).

However, it is not clear whether an inaccurate spatial representation affects viewers' liking or preference for one video over another, as filmmakers have suggested (e.g., Hochberg \& Brooks, 1996; Kraft et al., 1991). For instance, while 180-degree violations can affect spatial memory for items, it does not seem to affect people's memory for the narrative of a scene or the order of events within a scene (Frith \& Robson, 1975; Kraft et al., 1991) or even one's understanding of the narrative flow (Magliano \& Zacks, 2011).

Furthermore, an accurate spatial representation may only be important for the short duration of a single scene, and may not be remembered across the much longer duration of a movie (Hochberg \& Brooks, 1996; Levin \& Wang, 2009), suggesting that an accurate spatial representation may not be important to viewers in the long term. What is important is the viewer's experience of the film as seamless, with transitions between shots being perceived as smooth rather than jarring or confusing (Smith, 2012; see also Levin \& Wang, 2009).

There are a number of questions regarding the 180-degree rule that have not been put to the test: 
1. Do audiences notice violations of the 180-degree rule?

2. If violations are detectable, what are their effects on the subjective experience of the viewer? Specifically, do they cause confusion or disorientation, as has been proposed (Bordwell \& Thompson, 2012; Hochberg \& Brooks, 1996; Proferes, 2008; Shimamura, 2013)?

3. And by extension, do the violations detract from the filmic experience (Carroll, 1980)? The aim of the present investigation is to address each of these issues in turn.

\section{Experiment 1: Identification of Violations}

Audiences are worse at remembering where objects are located in a scene when 180-degree violations occur (Frith \& Robson, 1975; Kraft et al., 1991), which suggests that these violations impact spatial understanding of scenes (Huff \& Schwan, 2012). Viewers use common areas between two shots to understand the spatial layout of the scene (Germeys \& d'Ydewalle, 2007; d'Ydewalle et al., 1998; see also Levin \& Wang, 2009). Collectively this work implies that viewers are sensitive to 180 -degree violations. To our knowledge, no psychological research has directly addressed this issue (see Levin and Wang, 2009).

The aim of Experiment 1 was to assess the extent to which observers could identify 180degree violations. To ensure observers were identifying genuine violations, and to get a sense of the upper-limit of the detection rate, participants were given instruction as to what a 180-degree violation is and what it looks like. This is likely to overestimate the detection ability but aims to reduce the likelihood of mistakenly judging a cut to be a violation while also placing an upper bound on correct detection accuracy.

\section{Method}


Participants. Thirty-five undergraduate students (age: 18 to 26 years, $M=20.40,12$ males) participated, and were compensated with course credit. In all experiments, the sampling design was to collect a minimum of 30 participants per condition.

Materials. Twelve videos were shot and edited with the help of several UBC Film Production and Theatre students who were blind to the nature of the project. Each video featured two or three subjects in conversation with unique plots. The plots were comedic sketches between two or three people. A selection of the actors ( 2 male and 3 female actors) appeared across the 12 sketches. Royalty-free audio derived from Free Music Archive (2017) and YouTube (2017) was added to supplement dialogue. An example of a violated and non-violated video can be viewed at https://osf.io/3smdj/.

Two versions of each of the 12 videos were created. Each version differed only in two key shots. During the filming of each video, the cinematographer was asked to film two key moments from the side of the axis that followed the 180-degree rule, and then from the side that violated the rule. Actors were instructed to repeat their performance as closely as possible during each take, and the cinematographer was asked to match the shots so that the amount of time actors and items were on screen, and their relative locations were the same but flipped. This produced one version of each video with two 180-degree violations (denoted as violated), and one version of each video without any violations (denoted as non-violated). To make sure that there was no new information in the two versions of the shots, the preceding shots were made to include as much of the background and surroundings as possible, so that any differences in judgments are not the result of having seen different visual information. The average duration of 
the 12 sketches was edited to be 30 seconds long, and the average duration of a single shot was $2.50 \mathrm{~s}(S D=1.31 \mathrm{~s}) .^{2}$

Design and Procedure. Film shots, cuts, and violations of the 180-degree rule were defined and demonstrated with an example using a PowerPoint presentation. None of the example shots used in the instruction were taken from the stimulus set. The complete instructions for all three experiments can be found on the OSF page for this study: https://osf.io/3smdj/. To briefly explain, participants were given a small number of example images showing what a cut is and when it both does and does not violate the 180-degree rule.

Six videos containing 180-degree rule violations were selected randomly from the stimulus set once. The remaining six videos had no violations while ensuring that the content of these six videos differed to the content of the six videos containing the violating cuts. This randomization and pairing happened only once, creating the stimulus set for all participants. Participants were shown all 12 videos (six with violations, six without) in random order and instructed to press the spacebar whenever they noticed a violation of the 180-degree rule. These ratings were made in real-time, while watching the videos. Upon completion, the participants were debriefed.

\section{Results and Discussion}

These results address whether participants notice violations of the 180-degree rule (research question 1). Button presses that occurred after the violating cut but before the next cut were extracted. The same timeframe in the non-violated videos was used to extract button presses. The average duration of this critical period was $2.78 \mathrm{~s}(S D=1.32 \mathrm{~s})$. We compared the

\footnotetext{
2 The violated and non-violated versions were near identical in terms of overall duration and the duration of a single shot. For that reason, the means and standard deviations are only reported for the 12 non-violated videos to give a more accurate impression of the data distribution.
} 
responses to the violated versus non-violated versions at the point where the violation occurred (or would have occurred, in the non-violated videos) to determine if people detected violations. If viewers noticed the 180-degree rule violations, we would expect more responses around the violations in the violated videos than in the non-violated videos.

Participants responded to a proportional .42 of critical cuts in the violated videos, but only .08 of critical cuts in the non-violated videos, Table 1. A paired-samples Wilcoxon signed rank test revealed that lay viewers could distinguish 180-degree rule violations from non-violations, $Z$ $(34)=5.08, p<.001$. In other words, participants were roughly four times more likely to identify a critical cut as violating the rule if there really was a violation compared to when there was no violation.

Table 1. The median proportion of identification responses to critical and non-critical cuts in 180-degree violated and non-violated videos.

\begin{tabular}{|c|c|c|}
\hline & \multicolumn{2}{|c|}{$\begin{array}{c}\text { Median proportion of responses to cuts (interquartile } \\
\text { range) }\end{array}$} \\
\hline & Critical cuts & Non-critical cuts \\
\hline 180-degree violated videos & $.417(.208)$ & $.082(.102)$ \\
\hline Non-violated videos & $.083(.125)$ & $.063(.096)$ \\
\hline
\end{tabular}

It is important to consider that participants correctly identified only .42 of violated cues and did not respond to .58 of 180 -degree violations (i.e., 1-.42=.58) despite having received instruction about what a violation looks like. That they were given instruction on the 180-degree 
rule may suggest that the .42 detection rate is an overestimation, and that the error rate in detection may be greater than what was observed. It should be noted that participants mistakenly identified approximately one in 12 cuts as being a violation despite receiving instruction to detect 180-degree violations. In either case, more than half of the violated cuts were not detected under favorable conditions (i.e., after instruction on how to detect them), which suggests the negative impact on viewer's enjoyment may be small and/or infrequent, if at all noticeable. Experiments 2 and 3 explore this further. In these experiments, participants will not be given instruction about how to detect a violation in order to understand how violations affect the viewing experience without priming their detection.

The results of Experiment 1 suggest an answer to research question 1: Participants can identify 180-violations when they know what to look for, consistent with other work showing that viewers have a less accurate spatial understanding of the layout of a scene when a cut violates the 180-degree rule (Frith \& Robson, 1975; Huff \& Schwan, 2012; Kraft et al., 1991). However, even under these conditions participants miss a little more than half the violations and misidentify approximately one in 12 cuts as being violations, suggesting that there may be some difficulty with identifying violations even with instruction.

\section{Experiment 2: Disorienting Nature of Violations}

Having established that the 180 -violations in our films are detectable, we can now turn to the putative subjective impact these violations have on observers. Prior research suggests that cuts that violate the 180-degree rule may disorient viewers (Frith \& Robson, 1975; Kraft et al., 1991). Experiment 2 addresses this issue.

Precisely how the violations affect the viewer's enjoyment is unclear from the filmmaking literature, but professionals in the area have noted that violations lead to "the dynamics of the 
dramatic moment [being] broken" (Proferes, 2008). The rule is intended to "stabilize the space of the playing area so the spectator isn't confused or disoriented" (Giannetti, 2001). While researchers have focused on the effects of 180 violations on spatial understanding, Kraft et al. (1991) and Frith and Robson (1975) provided evidence of an effect on viewer's temporal and narrative understanding as well. That is, confusion and disorientation need not be confined to a spatial understanding, as alluded to by the broader strokes definitions offered by filmmakers quoted above. To be faithful to the filmmaking community's impression in the laboratory, we made use of the cognitive ethology framework (Kingstone, Smilek, \& Eastwood, 2008; Chisholm et al., 2014). According to cognitive ethology, the conceptual language used to describe human cognition and affect should, initially, be grounded in the concepts and language that are used by people in their everyday life, rather than conforming to those imposed on them by researchers. In this way, by beginning at the natural level, one's subsequent investigations are grounded in cognition and performance as it occurs in everyday life, and hence, one's lab-based findings are likely to scale up to a natural environment rather than being constrained to specific and controlled research environments and terminologies. Thus, we left it to our participants to define for themselves what is meant by 'confusing' and 'disorienting' with an aim to observe the underlying commonality to participants' interpretation

\section{Method}

Participants. Sixty-two undergraduate student participants (age: 17 to 29 years, $M=20.60,17$ males) took part and were compensated with course credit.

Design and Materials. The stimulus set and within-subjects design replicates Experiment 1: Participants viewed six videos with 180-degree violations and six without violations. 
Procedure. The procedure was similar to Experiment 1. Participants were shown 12 videos (six with violations, six without) one at a time. As in Experiment 1, participants were asked to press the spacebar in real-time to identify the cut they just viewed, but this time to identify the cut as one that was experienced as confusing or disorienting. Upon completion, participants were debriefed.

\section{Results and Discussion}

Research question 2 asks whether participants would more often identify the critical cuts those that crossed the 180-degree axis of action - as confusing or disorienting compared to nonviolated cuts at the same time point in the non-violated version of the video.

Table 2. The median proportion of cuts identified as confusing or disorienting in violated and non-violated videos.

\begin{tabular}{ccc}
\hline & \multicolumn{2}{c}{ Median proportion of responses to cuts (interquartile } \\
range) & & Non-critical cuts \\
\cline { 2 - 3 } 180-degree violated videos & $.083(.177)$ & $.037(.092)$ \\
Non-violated videos & $.063(.208)$ & $.049(.113)$ \\
\hline
\end{tabular}

In the violated condition, a proportional .08 of the critical cuts were tagged as confusing or disorienting while .06 of the same cuts in non-violated videos were tagged as confusing or disorienting, Table 2. A paired sample Wilcoxon signed rank test revealed that participants were more likely to interpret violated cuts as disorienting or confusing as compared to non-violated 
cuts, $Z(61)=2.26, p=.024$. One-sample Wilcoxon signed rank tests also found that the median identification rate for both the violated and non-violated videos were significantly greater than both zero (violated: $Z(61)=6.00, p<.001$; non-violated: $Z(61)=5.53, p<.001)$ and .05 (violated: $Z(61)=3.28, p=.001$; non-violated: $Z(61)=2.15, p=.032$ ). Though statistically significant, the size of the effect is relatively small. The effect, albeit small, is consistent with the findings of Frith and Robson (1975) and Kraft et al. (1991) that showed spatial understanding was affected by violation of the 180-degree rule. That is, the form of confusion or disorientation experienced could be a result of difficulties establishing a spatial understanding.

Thus, while people do seem to detect violations with a fairly substantial effect size (Experiment 1), that has a disproportionately small effect in terms of confusion or disorientation. Alternatively, given that participants in Experiment 1 were primed and given instruction on how to detect violations, and that participants here were not, it may be that these results give a more indirect reflection of the detection rate of 180-degree rule violations. In either case, the relatively low rate of confusion resulting from 180 -degree rule violations ( $8 \%$ versus $6 \%$ without violations) is surprising in the context of the filmmaking community's perception that, for example, the purpose of the 180-degree rule is to "stabilize the space of the playing area so the spectator isn't confused or disoriented" (Giannetti, 2001). In Experiment 3 we more directly assess the effect of violations on the viewing experience.

\section{Experiment 3: Preference for Non-Violations}

We have shown that the 180-degree rule violations are identifiable by viewers (Experiment 1), and that these cuts are more likely (albeit to a small degree) to lead viewers to report feeling disoriented and/or confused (Experiment 2). Our final experiment tackles research question 3 by examining whether viewers prefer sketches without violations over those with violations. 
Filmmakers have implied that violations of the 180-degree rule negatively impact the appreciation of film because of difficulties with building an accurate spatial representation (Bordwell \& Thompson, 2012; Hochberg \& Brooks, 1996; Proferes, 2008), although empirical evidence to support this position is lacking. Furthermore, Levin and Wang (2009) claim that expert filmmakers are more likely to violate the 180-degree rule compared to novices, which one may speculate is because experts intuit that violating cuts need not reduce viewer appreciation. This would imply that expert filmmakers are aware that violating 180-degree violations will not reduce viewing appreciation. Levin and Wang (2009) argue that experts explicitly violate the rule in order to make spatial information more or less salient to the viewer in a way that moves beyond rules of thumb such as the 180-degree rule. Thus while experts may violate the rule, it is suggested that the outcome - to maintain spatial understanding - is the same for both experts and novices. It is just that their methods differ. In Experiment 3 we consider whether viewers prefer videos that follow the 180-degree continuity rule over those that violate it. As in Experiment 2, we leave it to our participants to determine on what criteria they make their preference (see Kingstone et al., 2008) while applying a well-established controlled measure of choice preference, i.e., the two-alternative forced choice (2AFC) method (Fechner, 1860/1966).

Participants were shown a series of paired videos: one of the pair had a violating cut and the other (with a different story line) had a non-violating cut. In this way, we could assess if participants systematically prefer videos with the non-violations (i.e., participants judge that they prefer the non-violated video more than half the time).

\section{Method}

Participants. Ninety-two undergraduate student participants volunteered (age: 17 to 29 years, $M$ $=20.30,29$ males), and were compensated for their participation with course credit. 
Materials. Using the 12 video narratives from Experiment 1, six pairs were created. To create these pairs, 26 undergraduate raters were asked to view and rank-order the videos by using a computer mouse to arrange the shortcuts representing each video from least preferred (on the left of the screen) to most preferred video (on the right of the screen). The rank order for each video was recorded as a value between 1 (least preferred) and 12 (most preferred), Figure 1. After all raters had taken part, we paired videos such that the average difference in rank between paired videos was minimized. This resulted in the following pairings: Cookie $(M=5.34)$ with Butler $(M$ =5.50), Duckling $(M=5.81)$ with Teddy Bear $(M=5.96)$, Garbage $(M=6.00)$ with Magician $(M=6.00)$, Car Trunk $(M=6.11)$ with Tea Party $(M=6.15)$, Paper Plane $(M=.6 .92)$ with Guitar $(M=7.35)$, and Basketball $(M=7.92)$ with Mug $(M=8.92)$. These 26 raters did not take part in the following task.

Design and Procedure. To assess viewer preference we used the 2AFC procedure, which is a psychophysical method developed by Fechner (1860/1966) that allows one to quickly, effectively, and reliably measure the subjective experience of a person through their pattern of choices. Typically, an observer is presented with two alternative options, simultaneously or in quick succession, and asked to select the one that best represents their experience on a particular factor (e.g., the presence or absence of a target, the clarity of a visual image, or in our present case, a preference for one video over another). If there is a reliable subjective experience for one alternative versus the other, the probability of choosing one versus the other is significantly different. A simple everyday example of the 2AFC method occurs when a visual prescription for eyewear is determined. An observer is presented with different prescription levels, pitting one against the other (2AFC) and the observer states which prescription strength renders the clearest image. 

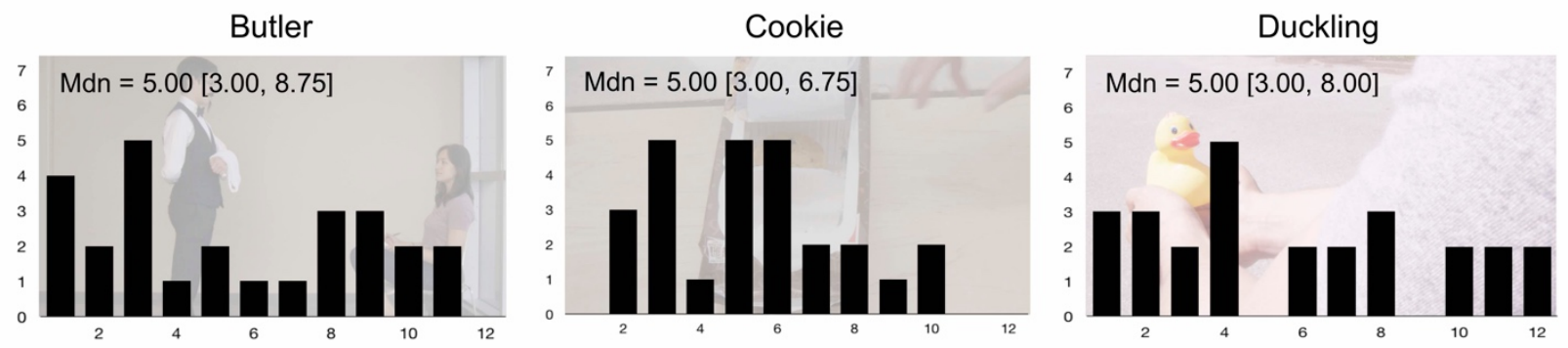

Tea Party

Teddy Bear

Car Trunk
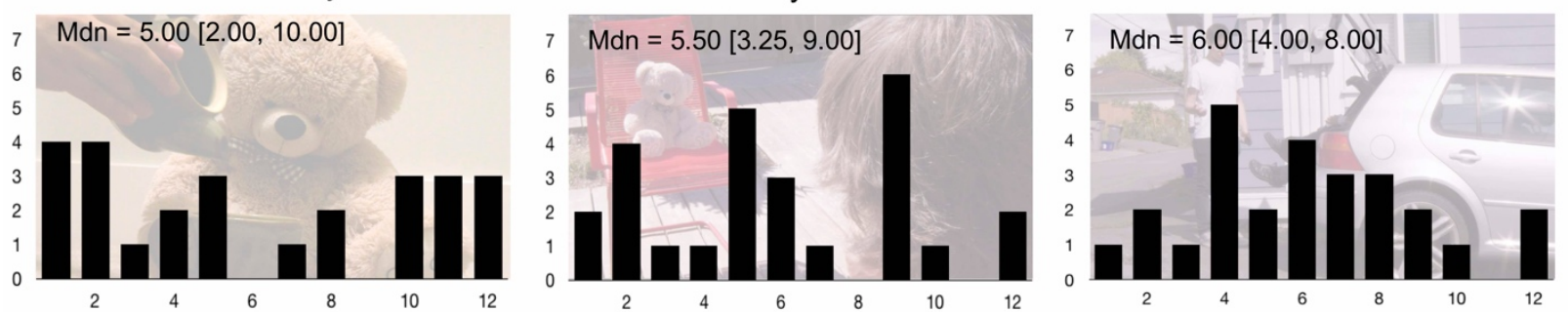

Garbage

Magician

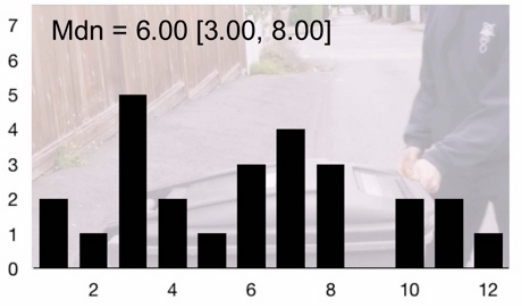

Paper Plane

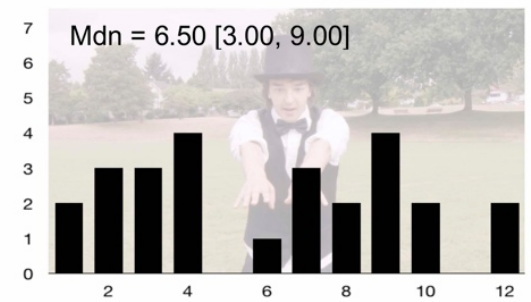

Guitar

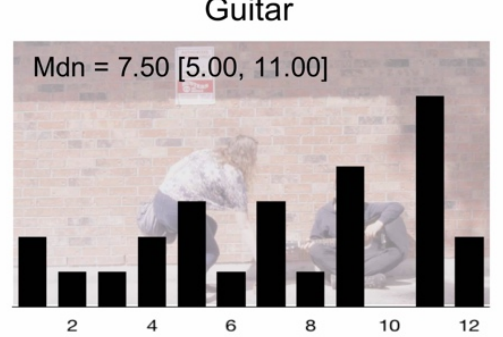

Basketball

Mug
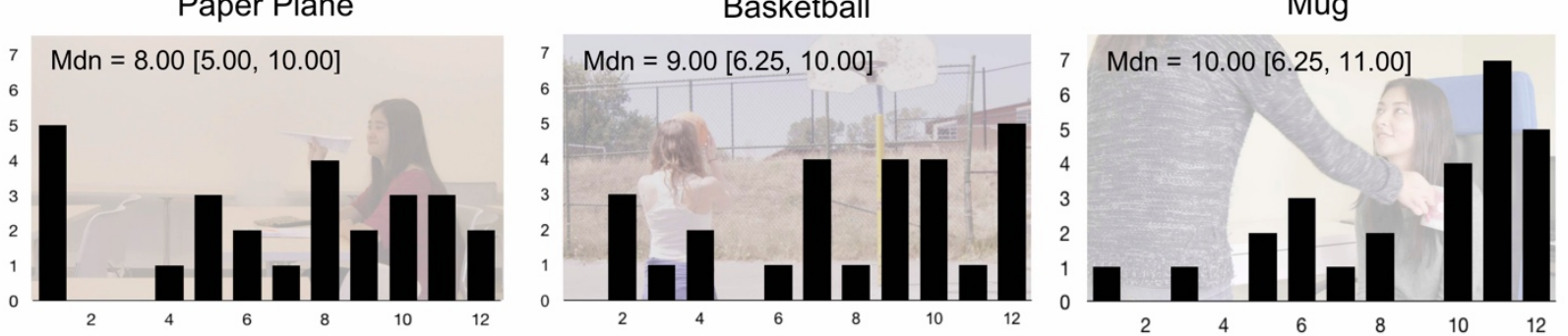

Figure 1 . The median rank $\left[25^{\text {th }}\right.$ percentile, $75^{\text {th }}$ percentile $]$ of each video and histogram of preference ratings, arranged from most (top-left) to least preferred (bottom-right). A rank value of one indicates the most preferred video, and a rank value of 12 indicates the least preferred video. 
Participants were randomly assigned to one of three video-pair conditions: (a) the nonviolated/non-violated pair in which neither video had any 180-degree violations (e.g., a 'Butler sketch' without a violation paired with a 'Cookie sketch' without a violation: $n=31$ ), intended to check for any viewing order effects (b) the non-violated/violated pair in which one of the videos contained violations (e.g., the Butler sketch with a violation paired with the Cookie sketch without a violation: $n=31$ ), and (c) the reciprocal violated/non-violated pair in which the other video in the pair contained violations (e.g., the Butler sketch without a violation paired with the Cookie sketch with a violation: $n=30$ ).

Participants viewed six pairs of videos and were asked to choose which video of each pair they preferred by pressing the left key (A) for the first video they saw, or the right key (L) for the second video they saw. Both the presentation order of the videos within each pair and the presentation order of each pair across the experiment were randomized. The video that participants identified as their preferred video was coded as a 1 and the non-preferred video coded as 0 . Next, an average preference for violated videos was calculated for each participant, which we refer to as the preference index. Averaged across trials, if violated videos were preferred equally as often as non-violated videos, a preference index of .50 would be recorded because on half of the trials the violated video would be preferred (trial coded as 1) and on half of the trials the non-violated video would be preferred (trial coded as 0 ). If participants preferred non-violated videos more often than violated videos, the preference index will be less than .50. A one-sample test demonstrating a preference index of significantly less than .50 would be evidence that non-violated videos were preferred over violated videos. For the condition where participants viewed pairs of non-violated videos, the proportion of participants who preferred the first video of the pair was coded as 1 and averaged across all trials for each participant. If 
participants systematically prefer the first or second video of a pair, a one-sample test would demonstrate evidence of a significant difference to a value of .50 .

\section{Results and Discussion}

Experiment 3 looked at whether violating the 180-degree rule in videos can cause the audience to be detracted from the filmic experience (research question 3).

There was no evidence to suggest that participants in the current study were more likely to prefer the first video presented in the pair compared to the second presented video when both videos contained no 180-degree violations (preference index $=.462, \mathrm{SD}=.186), t(30)=-1.13, p$ $=.269, d_{z}=-0.20 .^{3}$

Videos with violations were preferred over videos without a violation an average .519 proportion of the time $(S D=.222)$, where .50 would indicate an equal probability of preferring the violated and non-violated video. A one-sample $t$-test revealed that the proportion that preferred the violated videos was not statistically significantly greater than $.50, t(61)=0.68, p=$ $.700, d_{z}=0.09$.

The lack of evidence above for a statistical difference is not support for the claim that there is in fact no difference in violated vs. non-violated video preference. A Bayesian approach was used to address this issue, using the R package BayesFactor (Morey \& Rouder, 2013). A quick overview of the Bayesian approach used here can be found in the appendix. A JZS one-tailed one-sample $t$-test with a Cauchy prior width of $r=0.71$ yielded a Bayes factor that indicated a

\footnotetext{
${ }^{3}$ A Bayes factor was calculated to determine the relative evidence in favour of the claim that there was no order effect (i.e., the typical null position). A Bayes factor with a Cauchy prior scaled to $r=0.71$ indicated a moderate shift towards the belief that there were no order effects, $\mathrm{BF}=2.93$
} 
shift in belief from the alternative toward the null by a factor of 5.77. If one believed that the alternative hypothesis was as probable to be true as the null hypothesis in advance of collecting data, after having seen the data one should now believe the null of no effect of violations as being 6 times more likely than the alternative of there being an effect.

In summary, the results indicate that participants did not show a preference for videos without 180 -degree rule violations.

\section{General Discussion}

The 180-degree rule is used in filmmaking to help maintain continuity across shots. Film editors claim that violating the rule makes it difficult for viewers to develop an accurate spatial representation, which in turn detracts from viewers' experience of film (Hochberg \& Brooks, 1996; Kraft et al., 1991). We found that viewers recognize 180-degree violations (Experiment 1), and that these violations can be confusing and disorienting (Experiment 2). Notably, while we found a large effect size when investigating whether participants are able to spot violations ${ }^{4}$, we found a relatively small effect size when exploring whether they found those violations to be confusing and/or disorienting. Furthermore, violations did not cause a change in viewers' preference for a film (Experiment 3). Viewers liked videos with violations just as much as those without.

Continuity editing methods such as the 180-degree rule aim to maintain a sense that the next shot is occurring within the same time and space as the previous shot (Smith, 2012). Our findings are somewhat consistent with past studies that show that the 180-degree rule creates a sense of

\footnotetext{
${ }^{4}$ It is possible that some 180 -degree violations are not overtly detectable, but are covertly detectable. While this may be an interesting line of research to pursue, it is beyond the scope of the current research.
} 
spatial continuity and reduces viewer disorientation (Frith \& Robson, 1975; Kraft, 1987; Kraft et al., 1991; although see Germeys \& d'Ydewalle, 2007, for eye tracking evidence suggesting no disorientation as a result of 180 -degree violations).

Critically, however, and contrary to the received wisdom in filmmaking circles (Bordwell \& Thompson, 2012; Hochberg \& Brooks, 1996; Proferes, 2008), the present study found that videos were as likely to be preferred whether they contained a 180 -degree rule violation or not. This suggests that despite the fact that the violations can be detected under favorable conditions and that they produce some minor disorientation, they do not negatively impact a viewer's liking of the video. AToCC (Smith, 2012) may provide a framework to explain why spatial continuity does not affect viewing preferences. The theory argues that viewers do not construct a detailed spatiotemporal representation of the scene, and so spatiotemporal continuity is not critical to smooth continuity editing. There is a notable body of work showing that spatiotemporal representations can be detailed (Speed \& Vigliocco, 2014; Vinson, Engelen, Zwaan, Matlock, \& Dale, 2017; see also Mannaert, Dijkstra \& Zwaan, 2017), but in the context of film and media representations can be absent rather large changes (Levin, 2010; Levin \& Wang, 2009). Smith (2012, p.15) claims that:

'such effortful cognition is redundant for the perception of most important elements of a cinematic narrative. Editing a scene in a way that allows the perception of "continuity" is not about enabling the construction of a detailed spatiotemporal representation. Instead it is about enabling the viewer to shift their attention to the audiovisual details currently relevant to them and the narrative.' 
The detail of the spatiotemporal representation may prove an interesting line of inquiry for future theoretical work in the area.

The lack of a 180-degree rule violation effect may result from no such effect de facto existing. But it may also be that the enjoyment of the narratives in film negates the effect of a 180-degree rule violation. We chose to use the same narratives in both 180 -degree rule violated and non-violated videos, where the only difference between them was whether cuts crossed the axis of action or not, in order to attempt to control for the effect of narrative enjoyment and observe rule violation effects in isolation. No effect of 180-degree rule violations was observed between sketches with the same narratives. This dovetails with work on continuity editing (the art of combining clips in a way that give the appearance of a continuous narrative) which finds that cuts that create discontinuities in both time and action do not have a substantial effect on the viewer's understanding of the narrative (Magliano \& Zacks, 2011).

The results from Experiment 2 suggest that violations do lead to some confusion, although it is worth bearing in mind that the effect is small (participants reported approximately $8 \%$ of the critical cuts in violated videos as confusing versus $6 \%$ for the same cuts in non-violated videos). This low rate of confusion may result from many of the 180-degree rule violations passing unnoticed. Participants in Experiment 1 were given instruction on how to detect violations, and yet just over half of all violations went undetected. Although it is not possible to confirm with the current data, it may be that the low detection rate (even under the more optimal conditions of Experiment 1) is a result of change or edit blindness (Rensink, O’Regan \& Clark, 1997; Smith \& Henderson, 2008), where large changes in a scene or film edit go unnoticed, respectively. Because the camera's direction was the only change to result from the 180-degree violation manipulation, this suggests that the small effect on viewers' confusion or disorientation stems 
from changes in the spatial information. For instance, the present study controlled for the possibility that confusion resulting from violations of the 180-degree rule stem from novel background information by ensuring that the videos had establishing shots that introduced the entirety of the spatial layout. The study was not designed to identify why roughly $6 \%$ of nonviolated cuts were identified as confusing or disorienting, but could be related to any number of factors (e.g., the lack of speaking between the actors in some clips, the magic trick performed in some clips, the mildly comedic nature of the sketches which often played on unusual ideas). It may be worthwhile to explore the effect of film/television viewing frequency on perceptions of 180-degree rule violations. While this paper did not explore the frequency of viewing behavior, it may be that highly frequent viewing of non-violated cuts as portrayed on television and in films may lead to a surprise effect when a non-violated cut is detected. However, it is important to note that the effect size for disorientation or confusion in Experiment 2 was particularly small, and that even with instruction on how to detect violations participants failed to detect more than half of all violated cuts (Experiment 1).

In conclusion, 180-degree rule violations do not appear to negatively impact one's liking of videos, even though participants do consider these violations to be marginally more confusing and/or disorientating than non-violated versions of the same video. This suggests that the 180degree rule is not essential for maintaining smooth continuity. This may be because continuity editing is rooted in smoothly transitioning viewer's attention to elements relevant to the narrative moment. Filmmakers who wish to violate the 180-degree rule should do so with an understanding that, while it may cause a very small degree of disorientation, it does not detract from viewers' preference of a film. 
References

Ascher, S., \& Pincus, E. (2013). The filmmaker's handbook 2013: A comprehensive guide for the digital age. New York: Penguin.

Bordwell, D., \& Thompson, K. (2012). Film art: An introduction. New York: McGraw-Hill.

Carroll, J. M. (1980). Toward a structural psychology of cinema. New York: Walter de Gruyter. doi: $10.1515 / 9783110825619$.

Chisholm, J. D., Chapman, C. S., Amm, M., Bischof, W. F., Smilek, D., \& Kingstone, A. (2014). A cognitive ethology study of first- and third-person perspectives. PLoS One, 9(3): e92696. doi: 10.1371/journal.pone.0092696.

d'Ydewalle, G., Desmet, G., \& Van Rensbergen, J. (1998). Film perception: The processing of film cuts. In G. Understood (Ed.), Eye guidance in reading and scene perception (pp. 357367). London, UK: Elsevier. doi: 10.1016/b978-008043361-5/50017-1.

Fechner, G. T. (1966). In D. H. Howes \& E. C. Boring (Eds.), and H. E. Adler (Trans.), Elements of psychophysics. New York: Holt, Rinehart \& Winston. (Original work published 1860). doi: $10.1037 / 11304-026$.

Free Music Archive (2017). Retrieved from http://freemusicarchive.org/.

Frith, U., \& Robson, J. E. (1975). Perceiving the language of films. Perception, 4, 97-10. doi: 10.1068/p040097.

Germeys, F., \& d'Ydewalle, G. (2007). The psychology of film: Perceiving beyond the cut. Psychological Research, 71, 458-466. doi: 10.1007/s00426-005-0025-3.

Giannetti, L.D. (2001). Understanding movies. Upper Saddle River, NJ: Prentice Hall. 
Hochberg, J., \& Brooks, V. (1996). The perception of motion pictures. Cognitive ecology: Handbook of perception and cognition, 205-292. San Diego, CA: Academic Press. doi: 10.1016/b978-012161966-4/50008-6.

Huff, M., \& Schwan, S. (2012). Do not cross the line: Heuristic spatial updating in dynamic scenes. Psychonomic Bulletin \& Review, 19, 1065-1072. doi: 10.3758/s13423-012-0293-z.

Kingstone, A., Smilek, D., \& Eastwood, J. D. (2008). Cognitive ethology: A new approach for studying human cognition. British Journal of Psychology, 99, 317-340. doi: 10.1348/000712607X251243.

Kraft, R. N. (1986). The role of cutting in the evaluation and retention of film. Journal of Experimental Psychology: Learning, Memory, and Cognition, 12, 155-163. doi: 10.1037/0278-7393.12.1.155.

Kraft, R. N. (1987). Rules and strategies of visual narratives. Perceptual and Motor Skills, 64, 314. doi: $10.2466 /$ pms.1987.64.1.3.

Kraft, R. N., Cantor, P., \& Gottdiener, C. (1991). The coherence of visual narratives. Communication Research, 18, 601-616. doi: 10.1177/009365091018005002.

Levin, D. (2010). Spatial representation of the sets of familiar and unfamiliar television programs. Media Psychology, 13, 1-23. doi: 10.1080/15213260903563006.

Levin, D. T., \& Wang, C. (2009). Spatial representation in cognitive science and film. Projections: The Journal for Movies and Mind, 3, 24-52. doi: $10.3167 /$ proj.2009.030103

Magliano, J. P., \& Zacks, J. M. (2011). The impact of continuity editing in narrative film on event segmentation. Cognitive Science, 35(8), 1489-1517. doi: 10.1111/j.15516709.2011.01202.x. 
Mannaert, L. N. H., Dijkstra, K., \& Zwaan, R. A. (2017). Is color an integral part of a rich mental simulation? Memory and Cognition, 45, 974-982. doi: 10.3758/s13421-017-0708-1.

Mascelli, J. V. (1965). The five C's of cinematography. Hollywood, CA: Cine/Grafic Publications. doi: 10.1525/f1.1966.19.4.04a00320.

Morey, R. D., \& Rouder, J. (2013). BayesFactor: An R package for Bayesian analysis in common research designs. Retrieved from http://bayesfactorpcl.r-forge.r-project.org

Murch, W. (2001). In the blink of an eye: A perspective on film editing. Los Angeles, CA: Silman-James Press.

Proferes, N. T. (2008). Film directing fundamentals: See your film before shooting. Burlington, MA: Taylor \& Francis.

Rensink, R. A., O’Regan, J. K., \& Clark, J. J. (1997). To see or not to see: The need for attention to perceive changes in scenes. Psychological Science, 8, 368-373. doi: 10.1111/j.14679280.1997.tb00427.x.

Shimamura, A. P. (2013). Psychocinematics: Exploring cognition at the movies. Oxford: Oxford University Press. doi: 10.1093/acprof:oso/9780199862139.001.0001

Smith, T. J. (2012). The attentional theory of cinematic continuity. Projections, 6, 1-27. doi: 10.3167/proj.2012.060102.

Smith, T. J., \& Henderson, J. M. (2008). Edit blindness: The relationship between attention and global change blindness in dynamic scenes. Journal of Eye Movement Research, 2, 1-17. doi: 10.16910/jemr.2.2.6.

Speed, L. J., \& Vigliocco, G. (2014). Eye movements reveal the dynamic simulation of speed in language. Cognitive Science, 38, 367-382. doi: 10.1111/cogs.12096. 
Vinson, D. W., Engelen, J., Zwaan, R. A., Matlock, T., \& Dale, R. (2017). Implied motion language can influence visual spatial memory. Memory \& Cognition, 45, 852-862. doi: 10.3758/s13421-017-0699-y.

YouTube (2017). Retrieved from https://www.youtube.com. 


\section{Appendix: A Brief Overview of the Bayesian Approach to Difference Testing}

NHST gives us information about the probability of observing our collected data, assuming the null is true, the intentions of how much data to collect and when to stop, and other assumptions. The Bayesian approach can give probabilistic information about how to update our beliefs in the probability of a hypothesis, rather than giving information about the probability of observing data. Here we focus on the Bayes factor, which is the relative evidence in favor of the alternative hypothesis relative to the null.

A Cauchy distribution is used in this article to specify a prior belief about the hypothesis, i.e., a belief before seeing data. The scaling factor $r$ specifies how kurtotic the belief distribution is. For instance, $r=0.71$ means that we believe there is a $50 \%$ probability that the effect size ranges between $d=-0.71$ and $d=0.71$. We have chosen this weakly informative prior distribution and scaling factor because it is recommended for most experimental setups by Morey and Rouder (2013), it is readily computed, and gives a stable integration of the likelihood. The prior is weakly informative: it is not a subjective evaluation of the effect sizes in the literature, but rather a generalization of effect sizes that tend to be observed in the field of psychology.

The prior belief in the hypothesis can be updated by evidence. Multiplying the observed data by the prior belief distribution generates the posterior belief in the hypothesis (or rather, it does so after dividing by the probability of the data ${ }^{5}$ ). But we are working with a prior belief that

\footnotetext{
${ }^{5}$ Calculating the probability of observing the data can require sophisticated distribution sampling techniques not discussed here. In these cases, the posterior distribution is generated by running this sampler technique a given number of times (or iterations) in the hope of producing a stable result.
} 
is not necessarily a direct reflection of our beliefs in this particular effect. Instead, it is a reference prior that simplifies comparing our findings to other findings using the same reference prior. As such, we will not focus our interpretation on the posterior belief, but on the Bayes factor, which is the probability of observing the data under the alternative model compared to the null model. Values near one indicate that the probability of observing the data under each model is approximately equal.

One of the advantages of a Bayesian approach, and the primary reason for its use here, is that the Bayes factor can distinguish between a lack of evidence (Bayes factors of approximately 1.00) and evidence in favor of the null hypothesis of no difference (values that diverge from $1.00)$. 


\section{Author Biographies}

George V. Kachkovski is a nursing student at The University of British Columbia. He has previously completed his BA Hons. in Psychology, with a minor in Microbiology and Immunology.

Daniil Vasilyev is a graduate in film production and a master's student in the Department of Psychological Sciences, Birkbeck, University of London. He works as a researcher at the Mace Experimental Research Laboratories in Neuroscience (MERLiN) Lab, specializing in Cognitive Film Theory. His master's thesis is on the "Effects of Film Editing on Dynamic Attention ".

Michael Kuk is a master's student at the Centre of Industrial Relations and Human Resources, University of Toronto. He holds a Bachelor of Science in Behavioural Neuroscience.

Alan Kingstone (PhD, FRSC) is interested in the brain mechanisms of human behaviour in real world situations. He is a Professor and Distinguished Scholar in the Department of Psychology at The University of British Columbia.

Chris N. H. Street (PhD, FHEA) is a senior lecturer in the Department of Psychology, University of Huddersfield, and Director of the Social Conflict Lab (www.conflictlab.org). His work mainly explores how people decide whether others are lying or not. His twitter handle is @ cnhstreet. 